\title{
Root cone angle is enlarged in docs1 LRR- RLK mutants in rice
}

\author{
M. Bettembourg ${ }^{1,2}$, M. Dal-Soglio ${ }^{1,2}$, C. Bureau ${ }^{1,2}$, A. Vernet ${ }^{1,2}$, A. Dardoux ${ }^{1,2}$, M. Portefaix ${ }^{1,2}$, M. Bes ${ }^{1,2}$, D. Meynard ${ }^{1,2}$, \\ D. Mieulet ${ }^{1,2}$, B. Cayrol ${ }^{1,2}$, C. Perin ${ }^{1,2}$, B. Courtois ${ }^{1,2}$, J. F. Ma ${ }^{3}$ and A. Dievart ${ }^{1,2,4^{*}}$ (D)
}

\begin{abstract}
Background: The DEFECTIVE IN OUTER CELL LAYER SPECIFICATION 1 (DOCS1) gene belongs to the Leucine-Rich Repeat Receptor-Like Kinase (LRR-RLK) subfamily. It has been discovered few years ago in Oryza sativa (rice) in a screen to isolate mutants with defects in sensitivity to aluminum. The c68 (docs1-1) mutant possessed a nonsense mutation in the C-terminal part of the DOCS1 kinase domain.
\end{abstract}

Findings: We have generated a new loss-of-function mutation in the DOCS1 gene (docs1-2) using the CRISPR-Cas9 technology. This new loss-of-function mutant and docs1-1 present similar phenotypes suggesting the original docs 1-1 was a null allele. Besides the aluminum sensitivity phenotype, both docs 1 mutants shared also several root phenotypes described previously: less root hairs and mixed identities of the outer cell layers. Moreover, our new results suggest that DOCS1 could also play a role in root cap development. We hypothesized these docs1 root phenotypes may affect gravity responses. As expected, in seedlings, the early gravitropic response was delayed. Furthermore, at adult stage, the root gravitropic set angle of docs 1 mutants was also affected since docs 1 mutant plants displayed larger root cone angles.

Conclusions: All these observations add new insights into the DOCS1 gene function in gravitropic responses at several stages of plant development.

Keywords: Rice, Root cap, Angle, Gravitropism, docs1, Lrr, Rlk, GSA, RCA, RGA

\section{Findings}

Plant root systems show usually complex architectures, with most roots growing vertically (i.e. negative orthogravitropism) but some also growing more radially (i.e. plagiogravitropism) into the ground. The gravitropic equilibrium position at which organs will grow has been called the gravitropic set-point angle (GSA) (Digby and Firn 1995; Firn and Digby 1997). In fibrous root systems, like that of rice, the GSA of the shallowest crown roots is an important root parameter because it depicts well the global shape of the root system. To report this root system parameter, the root cone angle (RCA) or the root growth angle (RGA) are usually measured. The RCA is the angle between the two most external right and left roots in a two-dimensional system. The RGA is the angle between the soil surface and the shallowest crown

\footnotetext{
* Correspondence: anne.dievart@cirad.fr

'CIRAD, UMR AGAP, F34398 Montpellier, France

${ }^{2}$ AGAP, Univ Montpellier, CIRAD, INRA, Montpellier SupAgro, Montpellier, France Full list of author information is available at the end of the article
}

root (Kitomi et al. 2015). These two measures are then supplementary $\left(2 \mathrm{RGA}+\mathrm{RCA}=180^{\circ}\right)$. It is assumed that the degree of RCA openness or narrowness could have a strong effect on the ability of plants to explore their external surrounding to optimize their resource uptake (Lynch 2013; Uga et al. 2015). For example, an open RCA could impact the uptake efficiency of nutrient such as phosphate, which accumulates preferentially in the topsoil.

To date, two genes affecting RGA have been cloned in rice: DRO1 (DEEPER ROOTING 1) and SOR1 (SOILSURFACE ROOTING 1) (Hanzawa et al. 2013; Uga et al. 2011; Uga et al. 2013). Both genes could affect auxin signal transduction during the gravitropic response. This suggests that polar auxin transport during the gravitropic response is an important determinant of the RGA in rice plants, as it is in Arabidopsis. Indeed, it has been reported recently in Arabidopsis that regulation of polar auxin redistribution determined GSA in lateral roots (Rosquete et al. 2013). The high or low auxin level/signaling resulted in an axial or radial root system, respectively. Not surprisingly, the cellular 
and molecular mechanisms leading to auxin redistribution in cells and tissues for GSA establishment rely in part on the same actors than the ones involved in gravity response. First, gravity sensing depends on statoliths relocalization in columella cells. Then, relocalization of auxin efflux-carrier proteins on cell membranes will generate asymmetric, directional, specific and localized auxin flows across cells and tissues (Adamowski and Friml 2015). These polar auxin gradients will lead to differential root side cell expansion to activate root curvature (Armengot et al. 2016). The fine regulation of these spatio-temporal auxin fluxes will then be of great importance for root system architecture establishment and environmental adaptive responses.

The DEFECTIVE IN OUTER CELL LAYER SPECIFICATION 1 (DOCS1) gene belongs to the large family of leucine-rich repeat receptor-like kinases (LRR-RLKs) involved in many developmental and environmental responses (Wu et al. 2016). In rice, a mutant of this gene, named c68, carries a nonsense mutation in the kinase domain (Huang et al. 2012). The c68 mutant has been discovered in a screen for aluminum sensitivity (Huang et al. 2009). In aluminum-rich media, root elongation of c68 plants was inhibited compared to that of the Koshihikari wild type (WT) plants. The c68 mutant plants also showed several root phenotypes: a reduced number and size of root hairs, and layers of external tissues with exodermis/epidermis mixed identity (Huang et al. 2009).

In this study, we have created a null allele of DOCS1 and have shown that mutant plants exhibited impaired gravity responses at different developmental stages. At
3 days, a delay of response to gravity was observed during the first hour after gravistimulation. At 30 days, we observed that the RCA of mutant plants was more open than that of WT plants. Our new data suggest that the DOCS1 receptor could be involved in the auxin response at several stages of development. Root cap morphology and cell identity defects observed in several root external tissues may affect auxin efflux and influx transport in root tip following root gravistimulation, or at later stages during GSA establishment.

\section{Results}

\section{docs1-1 is a null allele}

In previous genetic analyses, the docs 1 mutant allele of c68, that will be renamed docs $1-1$ hereafter, has been shown to be recessive to the WT DOCS1 allele. This mutant allele possesses a 1-bp deletion in the tenth exon of the DOCS1 gene (LOC_Os02g14120, Os02g0236100), which could result in the production of a truncated receptor missing the end of the kinase domain (Huang et al. 2012). In our study, we wanted first to evaluate if gene activity was completely lost in the docs $1-1$ mutant. We therefore produced a new mutated allele of the DOCS1 gene by the CRISPR technology. We targeted this new mutation in the $5^{\prime}$ end of the DOCS1 cDNA (Fig. 1). The variant obtained presents a deletion of $1 \mathrm{bp}$ at nucleotide 96 after the start codon. This new mutated allele would be predicted to code for very short peptides of 39 amino acids corresponding to the first 32 amino acid of the DOCS1 protein plus 7 amino acids from out-

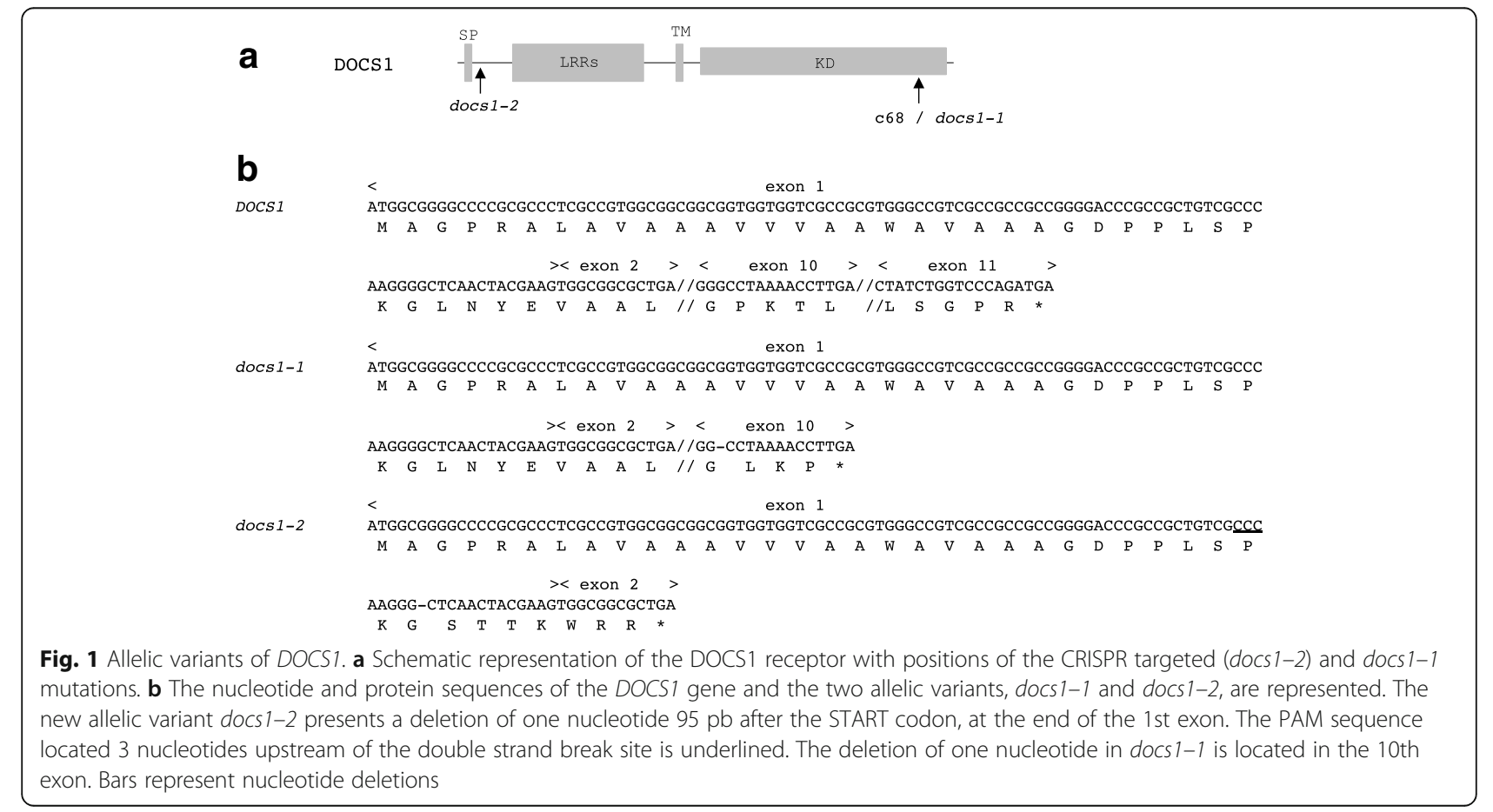


of-frame transcriptional residues. To evaluate if, like docs1-2, the docs1-1 allele was a loss-of-function, we compared docs1-1 and docs1-2 phenotypes. First, the root hair morphology of these mutant plants was observed under a binocular microscope. As seen previously for docs1-1 plants compared to their Koshihikari WT control (Huang et al. 2009; Huang et al. 2012), the docs1-2 mutant plants possessed less and shorter root hairs than their Nipponbare WT control (Fig. 2a-b). Second, we compared root anatomical parameters. Again, like in docs 1-1 plants, morphological changes of the outer cell layers were observed in the docs1-2 lines (Fig. 2c-d and Additional file 1: Figure S1). Indeed on root cross-sections $0.5 \mathrm{~cm}$ from the apex, and on polar views of these sections, epidermal, exodermal and sclerenchyma of mutant cells are not gathered in 3 well-defined layers. Even if some exodermal cells were identifiable among these disorganized tissues because of auto-fluorescence extinction at Casparian bands, most of the cells forming the root outer cell layers seemed to have mixed identities. Sometimes, the 3 sclerenchymal, exodermal and epidermal cell layers were identifiable among these disorganized tissues (see the white box in Fig. 2e), suggesting that some epidermis cells may have the ability to differentiate into root hair cell types. However, since these well-organized tissue areas are rare, it could explain why the root hairs on docs 1 mutant roots are so scarce. Since these root hair phenotypes have been shown to affect aluminum sensitivity in docs1-1 (Huang et al. 2009), we also tested the sensitivity to aluminum of the docs 1-2 lines. We used the protocol previously developed by Huang et al. (2009). After 5 days of growth in hydroponic solution, $30 \mu \mathrm{M}$ of $\mathrm{AlCl}_{3}$ was added to the media. The relative root elongation (RRE) of control and docs 1-2 mutant roots was calculated after a $24 \mathrm{~h}$ treatment. In concordance with what has been observed previously with docs1-1 plants, the docs1-2 line RRE was decreased of $86.5 \%$, in comparison with a reduction of only $66.7 \%$ for the WT Nipponbare control roots. These
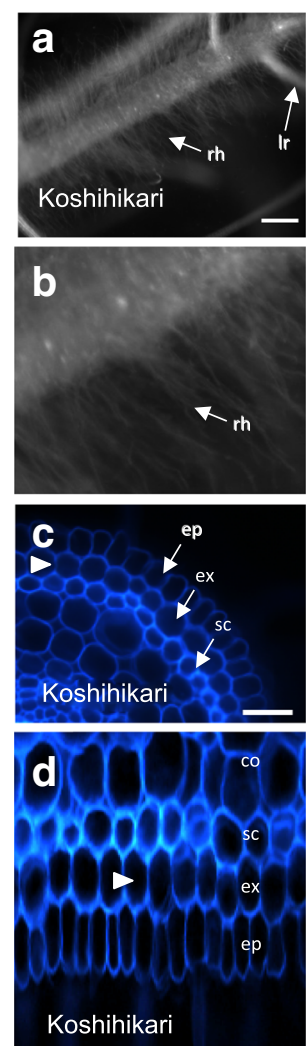
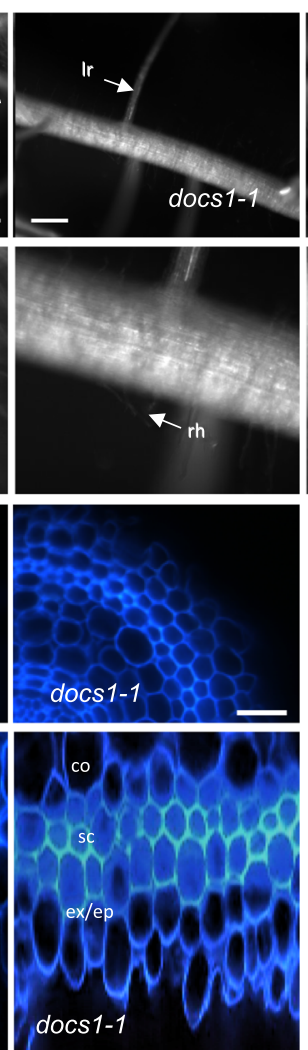
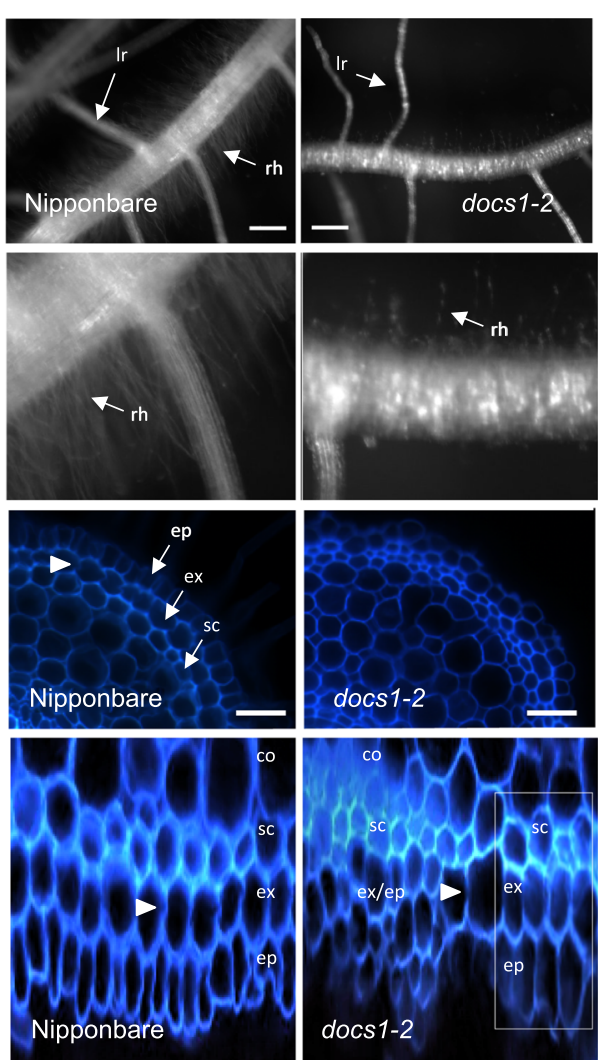

Fig. 2 docs 1-1 and docs 1-2 root mutant phenotypes compared to their respective Koshihikari and Nipponbare controls. a Root hairs of docs 1-1 and docs 1-2 mutant roots are much shorter and less abundant than their respective controls. Ir, lateral root; rh, root hairs. Bars $1 \mathrm{~mm}$. $\mathbf{b}$ Enlarged views of the root hair zones from (a). c Organization of outer root cell layers (epidermis (ep), exodermis (ex) and sclerenchyma (sc)) are affected in docs 1-1 and docs 1-2 mutant roots as observed on root cross-sections. The exodermis layer is characterized by a decreased of UV fluorescence on their radial cell walls due to Casparian strip presence (white arrowheads). Bars $20 \mu \mathrm{m}$. d Disorganization of outer layers is highlighted on polar views of the root cross-sections. Full images of these polar views made with the imageJ software (Lartaud et al. 2015) are displayed in Additional file 1: Figure S1. The white box in the docs 1-2 polar view highlights a zone where the three outer cell layers are less disorganized and where the exodermis and epidermis cell layers seem to have differentiated normally. co, cortex 
various phenotypic analyses show that the phenotypes observed for docs1-1 and docs1-2 mutant plants are identi$\mathrm{cal}$, indicating that the original docs1-1 allele is a loss-offunction mutation.

\section{docs 1 mutants show altered gravitropic perception and response at adult and seedling stages}

We hypothesized that the identity defects and disorganization of root outer cell layers in docs1 mutant roots could affect root gravitropism. We then grew control and docs 1 mutant plants in rhizoboxes for one month and compared several parameters of their root architectures (see M\&M for details). Consistently, larger crown RCA were measured on docs1 mutant plants than on their respective controls (Fig. 3a). In average, mutant RCA were $\sim 1.5$ larger than control ones $\left(\sim 60^{\circ}\right.$ vs. $\left.\sim 40^{\circ}\right)$ (Table 1). Since the rhizoscope phenotyping system consists in plexiglass sandwiches filled with glass beads, we wanted to exclude the possibility that the angle phenotype observed could be due to negative thigmotropism response differences between control and mutant plants. We then conducted a second experiment in which rhizoboxes were free of beads (data not shown). Again, mutant plants showed a larger RCA than WT plants, suggesting that gravitropic response more than thigmotropism was affected in docs1 mutant plants. To measure the gravitropic response, we grew docs 1 and control seedling plants on MS/2 solid medium in petri dishes. After 3 days of growth, petri dishes were rotated of $90^{\circ}$ and seminal root tip positions of each seedlings were recorded every hours (Fig. 3b). Results indicate that docs 1 mutants were less sensitive to gravistimulation than control plants. Indeed, docs 1 mutants responded less rapidly than their controls to gravistimulation. Within one hour, the angle formed by control plants was $\sim 35^{\circ}$ compared to $\sim 20^{\circ}$ for the docs 1 mutants. This marked difference of response was visible up to $10 \mathrm{~h}$ after gravistimulation. However, after $24 \mathrm{~h}$, both mutants and controls reached nearly the same angle of curvature. Looking at this response every $5 \mathrm{~min}$ for one hour revealed that this response difference was significant as early as $25 \mathrm{~min}$ after gravistimulation (Fig. 3c). While most control plants have already turned of $\sim 20^{\circ}$ less than $30 \mathrm{~min}$ after gravistimulation, docs1-1 mutant plants reached only $\sim 10^{\circ}$ in the same period of time. These data confirm that gravitropic perception and response are affected in docs 1 mutant plants and could explain why GSA is modified in these plants.
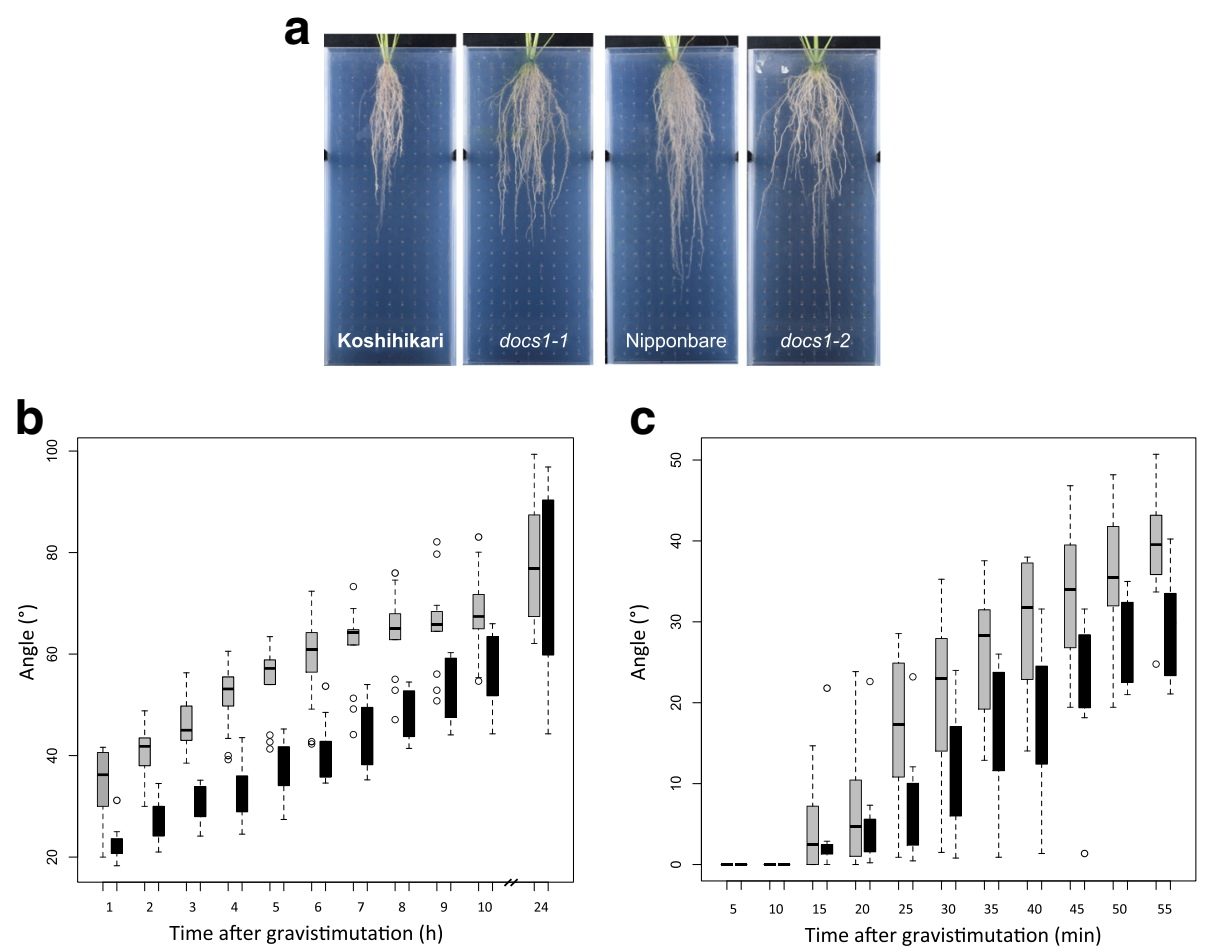

Fig. 3 docs mutants are affected in gravitropic responses. a At 28 days, docs 1 mutant plants grown under hydroponic conditions in rhizoboxes (plexiglas plates filled with $5 \mathrm{~mm}$ diameter glass beads, see M\&M for details) present a larger root cone angle than their respective controls. b, $\mathbf{c}$ Gravitropic response of docs 1-1 mutant (black) is affected compared to Nipponbare control (gray) seedlings. Seedlings have been grown for 3 days in the dark on MS/2 solid medium before rotating the petri dishes of $90^{\circ}$. Radicle angles have been measured every hour for 10 h, and at $24 \mathrm{~h}(\mathbf{b}, n=13$ for Koshihikari, $n=9$ for docs $1-1)$, and every 5 min for one hour $(\mathbf{c}, n=8$ for Koshihikari, $n=7$ for docs $1-1)$ 
Table 1 Root cone angle measures in WT and mutant plants grown in rhizoboxes

\begin{tabular}{llll}
\hline Plants & Angle $\left(^{\circ}\right)$ & SD & $\mathrm{n}$ \\
\hline Koshihikari & 40.1 & 6.1 & $>20$ \\
docs1-1 & 62.2 & 7.0 & $>20$ \\
Nipponbare & 43.2 & 6.4 & 6 \\
docs1-2 & 71.7 & 10.2 & 6 \\
\hline
\end{tabular}

\section{docs1 mutants present also root cap phenotypes}

Since gravity is primarily perceived by root caps, we then investigated the root cap anatomy and morphology of docs1 seminal roots (Fig. 4). First, we imaged longitudinal sections of 3 days old radicles under a multiphoton microscope (Fig. 4a). Previous study had already mentioned that lateral root caps were affected in docs $1-1$ mutants (Huang et al. 2012). We could confirm that in the docs 1 mutant plants, the lateral root cap cells were more tightly attached to the lateral root caps than in the WT. Moreover, besides this lateral phenotype, docs1 root cap tips seemed also shorter than WT. To go further into this analysis, we imaged radicle tips of embryos soaked in water for $24 \mathrm{~h}$ (Fig. 4b). This developmental stage has been used to avoid any bending artifact of the roots in the course of the experiments. At the embryonic stage observed here, docs 1 lateral root cap cells seem to desquamate on one side of the root cap leading to a stronger fluorescent signal. This phenotype is not observed on WT roots. Besides the lateral root cap detaching cells, docs1 mutant root cap shapes were also different from WT. Indeed, docs1 mutant root cap tips were shifted and not aligned on the root central radial axis. Moreover, columella cell files were bent in mutants. These anatomical and structural modifications of docs 1 mutant root cap cells suggest that the DOCS1 gene could play a role in root cap development during embryogenesis.

\section{Discussion}

Our data show that the loss of activity of the LRR-RLK DOCS1 gene affects gravitropism perception and response of rice roots at several stages of development. At seedling stage, our data on root cap developmental defect and external tissue disorganization suggest that the early gravitropic response, including gravity perception, could be impaired in docs1 mutants. At cellular and molecular levels, the timing of events leading to root bending after gravistimulation is well described in Arabidopsis, and rely on the formation of an asymmetrical lateral auxin gradient. This auxin differential flux
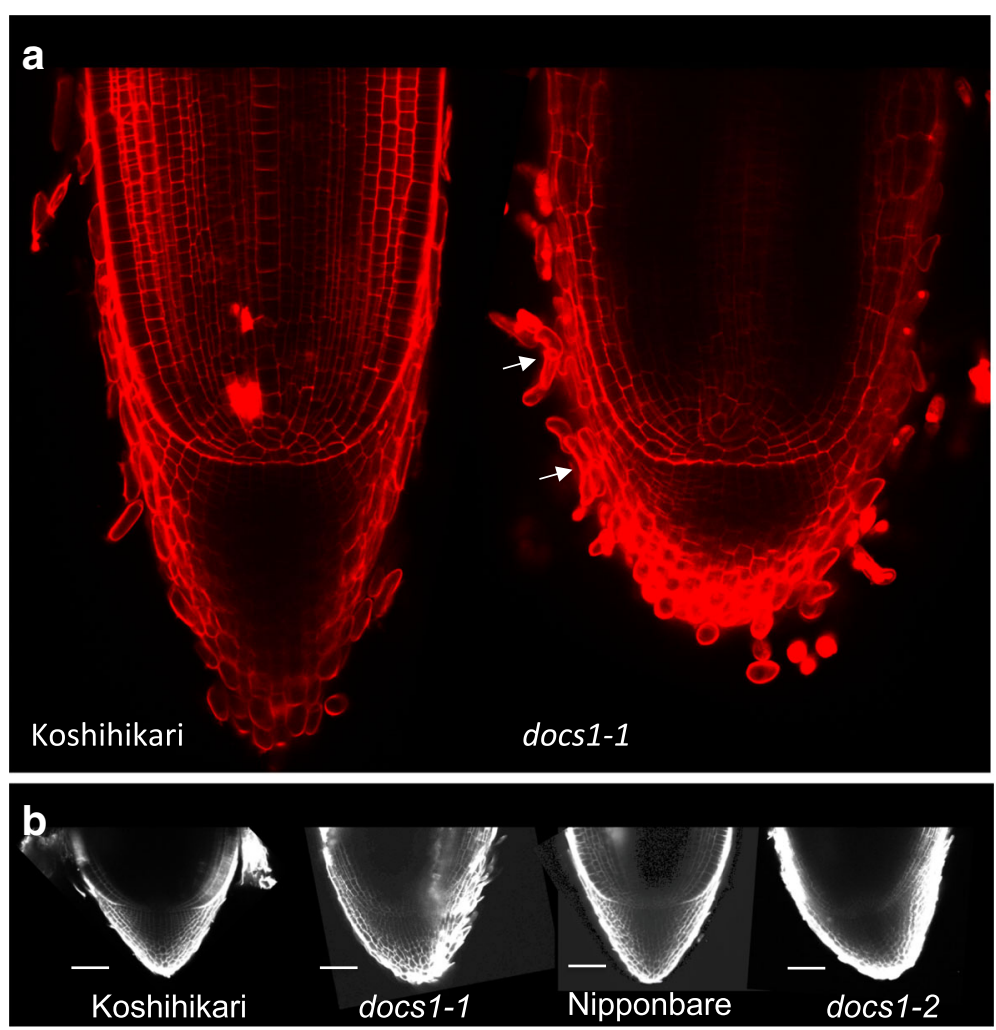

Fig. 4 Root cap phenotypes of docs 1 mutants stained with propidium iodide and imaged with a mutiphotonic microscope. a Longitudinal view of 3 days old radicle root tips. Tip of the docs 1 root cap seems shorter than control. Arrows point to lateral root cap cells which did not detach from other cells. b docs 1 embryo root caps are bent and shifted from the the root central radial axis. Bars $50 \mu \mathrm{m}$ 
between the upper and lower side of the root is initiated and maintained by polarized accumulation of several PIN proteins in lateral root cap and epidermis tissues. These differential lateral auxin gradients will activate root cell expansion on the upper side and inhibition of growth on the lower side, resulting in root bending (Band et al. 2012; Rosquete et al. 2013; Sato et al. 2015). In this way, the root cap and epidermal/exodermal morphological disorganizations observed in docs1 mutant roots could inhibit the early steps of lateral auxin gradient establishment.

In docs 1 mutant roots, molecular activation of the bending response could also be affected. The genomic answer, based on auxin-responsive gene expression, is observed $\sim 15 \mathrm{~min}$ after gravistimulation (Band et al. 2012; Sato et al. 2015). This genomic response relies on the regulation of several cell wall-related remodeling genes to trigger cell wall expansion on the upper side of the root to initiate bending. Previous transcriptomic data on docs $1-1$ plants have revealed that $1 / 3$ of the 60 genes that were misregulated more than 2-fold in the mutant roots compared to WT were genes involved in cell wall metabolism (Huang et al. 2012). Then, cell wall modifications required for root bending could also be perturbed in the docs 1 mutants.

The gravitropism defects observed in docs1 mutants lead to modifications of the GSA and result in the development of plant with more opened RCA. The RCA is an important trait in breeding programs, since it determines the soil volume that the plant explores and is potentially correlated to crop production under stress conditions (Uga et al. 2015). Nowadays, the discovery of new genes involved in this trait is then of particular interest to develop new varieties able to cope with changing environmental growth conditions. Radial expansion of the crown roots relies on the suppression of positive gravitropic responses. At cellular level, this response depends, like for gravistimulation response, on auxin redistribution fluxes that will induces differential root side growth. Thus, the same mechanisms responsible for the delayed gravity responses in seminal roots could also affect GSA establishment in crown roots. In docs1 mutant roots, a decrease in positive orthogravitropic response in crown roots would result in a more radial root system. This would again correspond to a lower polar auxin transport and/or signaling in these roots. However, if these two responses are comparable at the molecular level, the period of time involved in these two processes are different. Indeed, gravistimulated roots will respond within minutes when GSA will take several days to be settled.

To date, and based on our results, our understanding of the exact underlying mechanism of DOCS1 mediated gravity response is still incomplete. Indeed, each of the defects observed in docs1 mutant plants could participate in the gravity mutant phenotype, namely root cap development, outer root tissue differentiation, and/or misregulated cell wall-related genes. However and surprisingly, despite such morphological and molecular perturbations, docs1 mutant roots were still able to respond to gravity stimulation, even to a lesser extent. Another important point to take into consideration is that DOCS1 belongs to the SG_II LRR-RLK subfamily and possess 5 LRRs in its extracytoplasmic domain (Dufayard et al. 2017). Other 5LRR-containing receptors, like the SERKs or SOBIR, have been shown to be co-receptors for other LRR-RLK receptors (Ma et al. 2016). If DOCS1 is also a co-receptor, the different phenotypes observed could be related to its multiple partner functions. This hypothesis will have to be investigated in the future and further genetic and molecular analyses on docs 1 mutant roots will be needed to uncover the function of the DOCS1 gene in these processes.

\section{Methods \\ Plant materials}

The c68 (docs1-1) mutation, which is in a Koshihikari background, has been described previously (Huang et al. 2009; Huang et al. 2012). The new docs1 allele produced in this study by the CRISPR technology is in the Nipponbare background. The CRISPRsearch algorithm (www.genome.arizona.edu/crispr/CRISPRsearch.html) has been used to define the CRISPR target sequence in the DOCS1 gene (GGCACCTTCGTAGTTGAGCCCC TT). Bsal sites have been added to this sequence for gateway cloning (Invitrogen) into a pUBI-cas9 vector (Miao et al. 2013). Nipponbare calli transformation of this construct has been done through Agrobacterium tumefaciens-mediated stable transformation as previously described (Sallaud et al. 2004). Primary plant transformant DNA has been extracted using the standard MATAB protocol (Mieulet et al. 2013). Targeted region of the DOCS1 gene has been amplified by PCR with the Phusion TaqPolymerase (Thermo Scientific) and the following primers: AAAAAGCAGGCTTGGGG GACCCGCCGCTGTCG and TAACAAAATCCCACG AACGAATCAG. PCR products were sequenced and chromatograms were analyzed manually to identify mutations. The new mutant allele has been named docs1-2.

\section{Phenotyping \\ Root hairs and anatomy defects}

After sterilization, seeds were sown on a MS/2 solid medium in sterile petri dishes (Corning, 431.301; $20 \mathrm{~cm} \times$ $20 \mathrm{~cm}$ ). After six days of growth in a growth chamber (day/night temperature of $28 / 25{ }^{\circ} \mathrm{C}$ and a $12 \mathrm{~h}$ photoperiod, $500 \mu \mathrm{Em}-2 \mathrm{~s}^{-1}$ ), root seedlings were pictured under a binocular loop to observe root hair phenotypes. Root tips were also cut and embedded in liquid agarose (3\%) for sectioning. After solidification, roots were 
sectioned $(60 \mu \mathrm{m}$ thin slices) with an Hm650v Vibratome (Thermo Scientific Microm). Root sections were then transferred on glass slides humidified with distilled water. Observations were made with a LEICA DM4500 microscope. Epifluorescence was observed with a specific A cube (excitation in UV, excitation filter: BP 340-380, suppression filter: LP 425). For this experiment, at least two series of 5 seedlings of each genotype have been analyzed (docs1-1 vs. Koshihikari and docs1-2 vs. Nipponbare). For embryo root cap observations, after imbibition of dehusked dry seeds in water for $24 \mathrm{~h}$, embryos were excised from the endosperm with a razor blade. For longitudinal observations, seedlings were grown in sterile petri dishes on MS/2 solid medium for 3 days. Before observation, embryos and radicle root tips were put in $10 \mu \mathrm{M}$ propidium iodide for $1 \mathrm{~h}$ with occasional degassing. After rinsing briefly in water, root tip radicles of embryos and seedlings were observed and imaged with a Multi-photon Zeiss LSM 7MP OPO $(1100 \mathrm{~nm})$.

\section{Aluminum assay}

The protocol used has been previously described (Huang et al. 2009). Briefly, plants were germinated and cultivated in $5 \mathrm{l}$ containers on nets floating on a $0.5 \mathrm{mM} \mathrm{CaCl}$ solution $(\mathrm{pH} 4.5)$ in a growth chamber (12/12 day length $-21{ }^{\circ} \mathrm{C}$ night- $28{ }^{\circ} \mathrm{C}$ day) for 5 days. Eight containers have been used, each containing 40 seedlings (10 of each genotype analyzed). On day $5,30 \mu \mathrm{M}$ of $\mathrm{AlCl}_{3}$ hexahydrate solution were added to half the containers. Root length was measured on day 5 before the aluminum addition (excluding plants with very slow germination or stunted growth) and after $24 \mathrm{~h}$ of treatment. For both the control and the treated containers, the relative root elongation (RRE) was calculated ((root size after $\mathrm{AlCl}_{3}$ - root size before $\mathrm{AlCl}_{3}$ treatment) $\times 100$ )/ root size before $\mathrm{AlCl}_{3}$ treatment). Differences in RRE between the mutants and their respective WT were tested using the contrast method $(P<0.05)$ both for the control and the stressed conditions. The contrasts between the mutants and their respective WT were significant for the stressed conditions but not for the control conditions.

\section{Rhizoscope assay}

Seedlings were grown under hydroponic conditions in $50 \mathrm{~cm} \times 20 \mathrm{~cm} \times 3 \mathrm{~cm}$ plexiglas plates (rhizoboxes) filled or not with $5 \mathrm{~mm}$ diameter glass beads in the rhizoscope platform as described in detail in (Bettembourg et al. 2017; Courtois et al. 2013). Each rhizobox contained a grid of nails, which held the root system in place after bead removal. After 28 days of growth, several parameters were measured (number of tillers, length of the longest leaf, maximum root length, depth reached by at least three crown roots and angle of the root cone). A first experiment has been conducted to compare docs $1-1$
( $>20$ plants) to their WT Koshihikari control (>20 plants), and docs1-2 (6 plants) to their Nipponbare control (6 plants). In a second experiment, rhizoboxes with and without beads were used. In this experiment, six docs1-1 and six WT Koshihikari control plants were phenotyped for each treatment.

\section{Gravitropic assay}

Seedlings were grown on a MS/2 solid medium in sterile petri dishes for 3 days in the dark. Then, a 90 degreerotation was performed on the petri dishes. In two different experiments, the radicle tip positions were marked (i) every $5 \mathrm{~min}$ for one hour, or (ii) every hour for $10 \mathrm{~h}$ and at $24 \mathrm{~h}$. At the end of the experiments, the plates were scanned. The scanned images were then analyzed with ImageJ to measure the angle of the root tip relative to the original position at t0. Three experiments with $\sim 10$ seedlings of each genotype have been performed. Seedlings whose roots did not response in the first hour of the experiments have been discarded from the analysis.

\section{Additional file}

Additional file 1: Figure S1. Polar views of docs 1 root cross sections made with the imageJ software. Only outer root cell layers (epidermis (ep), exodermis (ex) and sclerenchyma (sc)) are affected in docs 1-1 and docs 1-2 mutant roots compared to their respective Koshihikari and Nipponbare controls. No difference is observed in other tissues like endodermis (en) or cortex (co). The white box in the docs 1-2 polar view highlights a zone where the three outer cell layers are not disorganized. (JPEG 367 kb)

\section{Abbreviations}

CRISPR: Clustered Regularly-Interspaced Short Palindromic Repeats;

DOCS1: Defective In Outer Cell Layer Specification 1; GSA: Gravitropic Setpoint Angle; LRR-RLK: Leucine-Rich Repeat Receptor-Like Kinase;

PAM: Protospacer Adjacent Motif; RCA: Root Cone Angle; RGA: Root Growth Angle; RRE: Relative Root Elongation; WT: Wild Type

\section{Acknowledgements}

The technical assistance of C. Chaine, R. Michel and E. Lorenzini for greenhouse supports is greatly acknowledged. Authors thank the MRI-PHIVLAVALETTE (Montpellier RIO Imaging - Histocytology and Plant Cell Imaging Platform) for the imaging facilities and the staff for their technical support.

\section{Funding}

This work was supported by the Centre de coopération Internationale de Recherche en Agronomie pour le Développement (CIRAD) PhD fellowship to MBet.

\section{Authors' contributions}

JFM provided docs $1-1$ seeds. MBet, $C P, B C o$ and ADi designed the experiments. MBet, MD-S and CB performed the root anatomy phenotypings. MBet and MD-S performed the aluminum assays. MBet, $A D a$ and $B C o$ performed the rhizoscope assays with the help of CB, MP, MBes, BCa and ADi. MBet and CB performed the gravitropic assays. MBet, $A V$ and MP performed the rice transformation with the help of MBes, BCa, DMe and DMi. MBet and ADi wroted the manuscript with the help of BCO, CP and JFM. All authors read and approved the final manuscript.

Consent for publication

Not applicable

Competing interests

The authors declare that they have no competing interests. 


\section{Publisher's Note}

Springer Nature remains neutral with regard to jurisdictional claims in published maps and institutional affiliations.

\section{Author details}

${ }^{1}$ CIRAD, UMR AGAP, F34398 Montpellier, France. ${ }^{2}$ AGAP, Univ Montpellier, CIRAD, INRA, Montpellier SupAgro, Montpellier, France. ${ }^{3}$ Institute of Plant Science and Resources, Okayama University, Chuo 2-20-1, Kurashiki 710-0046, Japan. ${ }^{4}$ Present address: Shanghai Jiao Tong University (SJTU), School of Life Sciences and Biotechnology, Shanghai 200240, China.

Received: 25 May 2017 Accepted: 30 November 2017

Published online: 15 December 2017

\section{References}

Adamowski M, Friml J (2015) PIN-dependent auxin transport: action, regulation, and evolution. Plant Cell 27:20-32

Armengot L, Marquès-Bueno MM, Jaillais Y (2016) Regulation of polar auxin transport by protein and lipid kinases. J Exp Bot 67:4015-4037

Band LR, Wells DM, Larrieu A, Sun J, Middleton AM, French AP, Brunoud G, Sato EM, Wilson MH, Péret B, Oliva M, Swarup R, Sairanen I, Parry G, Ljung K, Beeckman T, Garibaldi JM, Estelle M, Owen MR, Vissenberg K, Hodgman TC, Pridmore TP, King JR, Vernoux T, Bennett MJ (2012) Root gravitropism is regulated by a transient lateral auxin gradient controlled by a tipping-point mechanism. Proc Natl Acad Sci U S A 109:4668-4673

Bettembourg M, Dardou A, Audebert A, Thomas E, Frouin J, Guiderdoni E, Ahmadi N, Perin C, Dievart A, Courtois B (2017) Genome-wide association mapping for root cone angle in rice. Rice (N Y) 10:45

Courtois B, Audebert A, Dardou A, Roques S, Ghneim-Herrera T, Droc G, Frouin J, Rouan L, Goze E, Kilian A, Ahmadi N, Dingkuhn M (2013) Genome-wide association mapping of root traits in a japonica rice panel. PLoS One 8:e78037

Digby J, Firn R (1995) The gravitropic set-point angle (GSA): the identification of an important developmentally controlled variable governing plant architecture. Plant Cell Environ 18:1434-1440

Dufayard JF, Bettembourg M, Fischer I, Droc G, Guiderdoni E, Perin C, Chantret N, Dievart A (2017) New insights on leucine-rich repeats receptor-like kinase orthologous relationships in angiosperms. Front Plant Sci 8:381

Firn RD, Digby J (1997) Solving the puzzle of gravitropism—has a lost piece been found? Planta 203:S159-S163

Hanzawa E, Sasaki K, Nagai S, Obara M, Fukuta Y, Uga Y, Miyao A, Hirochika H, Higashitani A, Maekawa M, Sato T (2013) Isolation of a novel mutant gene for soil-surface rooting in rice (Oryza Sativa L.) Rice (N Y) 6:30

Huang CF, Yamaji N, Nishimura M, Tajima S, Ma JF (2009) A rice mutant sensitive to al toxicity is defective in the specification of root outer cell layers. Plant Cell Physiol 50:976-985

Huang CF, Yamaji N, Ono K, Ma JF (2012) A leucine-rich repeat receptor-like kinase gene is involved in the specification of outer cell layers in rice roots. Plant J 69:565-576

Kitomi Y, Kanno N, Kawai S, Mizubayashi T, Fukuoka S, Uga Y (2015) QTLs underlying natural variation of root growth angle among rice cultivars with the same functional allele of DEEPER ROOTING 1. Rice (N Y) 8:16

Lartaud M, Perin C, Courtois B, Thomas E, Henry S, Bettembourg M, Divol F, Lanau N, Artus F, Bureau C, Verdeil JL, Sarah G, Guiderdoni E, Dievart A (2015) PHIV-RootCell: a supervised image analysis tool for rice root anatomical parameter quantification. Front Plant Sci 5:790

Lynch JP (2013) Steep, cheap and deep: an ideotype to optimize water and N acquisition by maize root systems. Ann Bot 112:347-357

Ma X, Xu G, He P, Shan L (2016) SERKing Coreceptors for receptors. Trends Plant Sci 21:1017-1033

Miao J, Guo D, Zhang J, Huang Q, Qin G, Zhang X, Wan J, Gu H, LJ Q (2013) Targeted mutagenesis in rice using CRISPR-Cas system. Cell Res 23:1233-1236

Mieulet D, Dievart A, Droc G, Lanau N, Guiderdoni E (2013) Reverse genetics in rice using Tos17. Methods Mol Biol 1057:205-221

Rosquete MR, von Wangenheim D, Marhavý P, Barbez E, Stelzer EH, Benková E, Maizel A, Kleine-Vehn J (2013) An auxin transport mechanism restricts positive orthogravitropism in lateral roots. Curr Biol 23:817-822

Sallaud C, Gay C, Larmande P, Bes M, Piffanelli P, Piegu B, Droc G, Regad F, Bourgeois E, Meynard D, Perin C, Sabau X, Ghesquiere A, Glaszmann JC, Delseny M, Guiderdoni E (2004) High throughput T-DNA insertion mutagenesis in rice: a first step towards in silico reverse genetics. Plant J 39:450-464
Sato EM, Hijazi H, Bennett MJ, Vissenberg K, Swarup R (2015) New insights into root gravitropic signalling. J Exp Bot 66:2155-2165

Uga Y, Kitomi Y, Ishikawa S, Yano M (2015) Genetic improvement for root growth angle to enhance crop production. Breed Sci 65:111-119

Uga Y, Okuno K, Yano M (2011) Drol, a major QTL involved in deep rooting of rice under upland field conditions. J Exp Bot 62:2485-2494

Uga Y, Sugimoto K, Ogawa S, Rane J, Ishitani M, Hara N, Kitomi Y, Inukai Y, Ono K, Kanno N, Inoue H, Takehisa H, Motoyama R, Nagamura Y, Wu J, Matsumoto T, Takai T, Okuno K, Yano M (2013) Control of root system architecture by DEEPER ROOTING 1 increases rice yield under drought conditions. Nat Genet 45:1097-1102

Wu Y, Xun Q, Guo Y, Zhang J, Cheng K, Shi T, He K, Hou S, Gou X, Li J (2016) Genome-wide expression pattern analyses of the Arabidopsis leucine-rich repeat receptor-like kinases. Mol Plant 9:289-300

\section{Submit your manuscript to a SpringerOpen ${ }^{\circ}$ journal and benefit from:}

- Convenient online submission

- Rigorous peer review

- Open access: articles freely available online

- High visibility within the field

- Retaining the copyright to your article

Submit your next manuscript at $\gg$ springeropen.com 